\title{
COMBINED THORACOSCOPIC/LAPAROSCOPIC STAGING OF ESOPHAGEAL CANCER
}

Mark J. Krasna, MD ${ }^{\text {a§ }}$

John L. Flowers, MD ${ }^{\text {b }}$

Safuh Attar, MD

Joseph McLaughlin, MD
Unlike mediastinoscopy in lung cancer, there exists no standard minimally invasive test to stage esophageal cancer. If it were possible to obtain exact preoperative staging in esophageal cancer, patients could be separated prospectively to receive neoadjuvant therapy appropriately. We studied the feasibility and efficacy of thoracoscopic and laparoscopic lymph node staging in esophageal cancer. Thoracoscopic staging was performed in 45 patients with biopsy-proven carcinoma of the esophagus. Laparoscopic staging was done in the last 19 patients. Thoracoscopic staging was aborted in three patients because of adhesions. Thoracic lymph node stage was N0 in 39 patients and N1 in three; celiac lymph nodes were normal in 13 and diseased in six. Esophageal resection was performed in 30 patients after thoracoscopic staging; 17 of these underwent laparoscopic staging. Thoracoscopic staging showed NO lymph node status in 28 patients and $\mathrm{N} 1$ in two patients. Two of the 28 patients (7\%) with N0 disease were found at resection to have paraesophageal lymph node involvement (N1); thus the disease was understaged by thoracoscopic staging. Thoracoscopic staging was accurate in detecting the presence of diseased thoracic lymph nodes in 28 of 30 cases (93\%). Laparoscopic staging detected normal celiac nodes in 12 patients and diseased lymph nodes in five patients. After esophagectomy, the final pathology report in the 12 patients with N0 disease was $\mathrm{NO}$ in 11 and diseased lymph nodes in one patient. Thus laparoscopic staging was accurate in detecting lymph node metastases in 16 of 17 patients $(94 \%)$. Thoracoscopic and laparoscopic staging are more accurate than existing staging methods. Six of 19 patients in whom laparoscopic staging was used had unsuspected celiac axis lymph node involvement that had been missed by standard noninvasive techniques. One of three patients with thoracic lymph nodes and three of six with celiac lymph nodes were downstaged after preoperative chemotherapy/radiotherapy. The role of thoracoscopy and laparoscopy in staging esophageal cancer should be further evaluated in a multiinstitutional trial. (J THORAC CARDIOvasc SuRg 1996;111:800-7)
$E_{\text {itions }}^{\text {sing }}$ ophageal cancer treated by surgery alone continues to have a dismal prognosis. Recent therapeutic protocols including chemotherapy and radia-

From the Divisions of Thoracic and Cardiovascular Surgery ${ }^{\mathrm{a}}$ and General Surgery, ${ }^{b}$ Department of Surgery, University of Maryland School of Medicine, Baltimore, Md.

Read at the Seventy-fifth Annual Meeting of The American Association for Thoracic Surgery, Boston, Mass., April 23-26, 1995.

Received for publication April 27, 1995; revisions requested July 7, 1995; revisions received Oct. 9, 1995; accepted for publication Oct. 31, 1995.

Address for reprints: Mark J. Krasna, MD, Division of Thoracic Surgery, Box 167, University of Maryland Medical System, 22 S. Greene St. Rm. N4W87, Baltimore, MD 21201.

${ }^{\S}$ By invitation.

Copyright (C) 1996 by Mosby-Year Book, Inc.

$0022-5223 / 96 \$ 5.00+0 \quad \mathbf{1 2 / 6 / 7 0 5 9 6}$ tion therapy have yielded improved survival results although associated with significant morbidity. ${ }^{1}$ Accurate preoperative lymph node staging would identify those patients who may benefit from neoadjuvant radiation or chemotherapy before esophagectomy and spare unnecessary morbidity to those who would not benefit. Pretreatment staging would also allow surgeons to assess the effectiveness of preoperative treatment and document pathologic downstaging when this occurs.

Thoracoscopic staging has been shown to accurately assess esophageal cancer by sampling thoracic lymph nodes $(\mathrm{N})$ and evaluating tumor primary $(\mathrm{T})$ status. $^{2}$ Because of a $7 \%$ incidence of unsuspected celiac lymph node metastases in our preliminary experience, ${ }^{3}$ routine laparoscopic exploration with celiac and lesser curvature lymph node sampling has been added to the standard thoracoscopic staging protocol. 
The objective of this study was to evaluate the feasibility, morbidity, and mortality of preoperative staging by thoracoscopy and laparoscopy for esophageal cancer. Accuracy of lymph node staging by thoracoscopy and laparoscopy in comparison with final pathologic staging after esophageal resection was assessed. The operative technique of staging thoracoscopy and laparoscopy is described in detail.

\section{Patients and methods}

All patients with histologically documented squamous cell carcinoma or adenocarcinoma of the thoracic (below $20 \mathrm{~cm}$ ) esophagus or gastroesophageal junction referred to the University of Maryland Medical Center or the Baltimore Veterans Affairs Hospital from November 1992 to November 1994 were evaluated. Patient work-up included esophagoscopy with biopsy, bronchoscopy for lesions adjacent to the carina or main-stem bronchi, conventional computed tomographic (CT) scan of the chest and upper part of the abdomen, magnetic resonance imaging (MRI), and esophageal ultrasonography (EUS). Patients with distant metastases or obvious local invasion of adjacent structures (including aorta, tracheobronchial tree, heart, mediastinum) on the basis or preoperative evaluation were excluded from further minimally invasive evaluation.

All laparoscopic lymph node staging procedures were performed by one (J.L.F.) of two general surgeons or the same thoracic surgeon. Correlation between operative findings at lymph node staging and resection were noted and pathologic specimens from minimally invasive staging and esophagectomy were compared. Lymph node samples were labeled according to esophageal cancer lymph node map terminology as described by Casson and associates. ${ }^{4}$

Technique of thoracoscopic lymph node staging. Right thoracoscopy was performed using one-lung ventilation with the patient in left lateral decubitus position. One monitor was placed over the patient's head and the other over the legs. This allowed both surgeon and assistant to look in the same direction and avoided "mirror imaging." The telescope was introduced into the sixth intercostal space at the posterior axillary line. Two additional trocars were inserted under direct vision. With the use of carbon dioxide insufflation ${ }^{5}$ and adjustment of the patient's position to take advantage of gravity, the lung was collapsed and allowed to fall out of the surgical field. A fourth trocar was occasionally inserted in the third intercostal space at the anterior axillary line, thus allowing three anterior trocars and one posterior trocar in a so-called "baseball diamond" configuration (Fig. 1). Alternatively, an operating telescope was used in most of the recent cases, obviating the need for a fourth trocar.

The mediastinal pleura overlying the upper part of the esophagus was incised and the incision was continued inferiorly to the level of the azygos vein down to the subcarinal region and finally to the inferior pulmonary vein. The tracheoesophageal groove was dissected, and biopsy specimens of the lymph nodes were obtained with blunt dissection. Surgical clips were used for hemostasis (Fig. 2). The area of the tumor was routinely

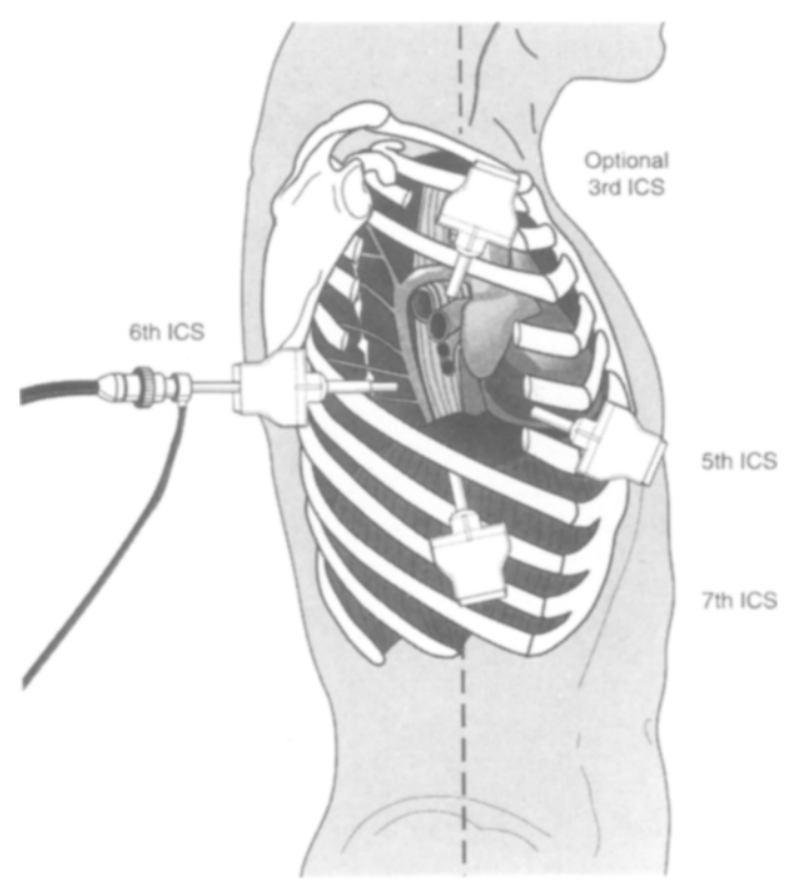

Fig. 1. Thoracoscopic positioning and port placement for right thoracoscopic lymph node dissection. ICS, Intercostal space.

inspected for evidence of direct invasion into surrounding structures.

Left thoracoscopy was performed if the CT scan, MRI, or EUS was suggestive of aortopulmonary or periaortic lymph node enlargement. The patient was placed in the right lateral decubitus position, and single-lung ventilation was accomplished. An incision was made along the posterior axillary line in the fifth intercostal space. Two additional incisions were made in the fourth intercostal space in the midclavicular line and in the seventh intercostal space in the anterior axillary line (Fig. 3). The mediastinal pleura overlying the aortopulmonary window was elevated with a biopsy forceps. The incision was continued cephalad up to the apex of the triangle formed by the phrenic and vagus nerves. Inferiorly, the pleura was incised over the left main pulmonary artery. Lymph nodes in the region were mobilized with a Babcock or Allis grasper, and the vascular pedicle was ligated with an endoscopic surgical clip as the node was held on traction.

Technique of laparoscopic lymph node staging. The patient was placed in the supine or modified lithotomy position, with the surgeon standing on the patient's left or between the legs, respectively. Both monitors were set at the head of the table. The abdomen was prepared and draped as for a standard laparotomy. The procedure was begun with three $10 \mathrm{~mm}$ diameter operating ports, although a fourth port was occasionally necessary in the left upper quadrant for retraction of the stomach or placement of tension on the hepatogastric ligament (lesser omentum) (Fig. 4). An angled laparoscope (30 degrees or greater) was essential for exposure of the operative field. 


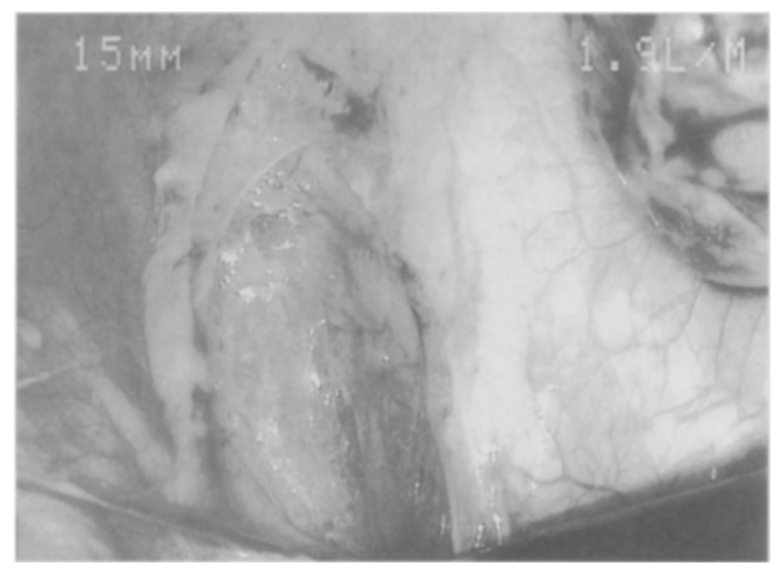

Fig. 2. Dissection of the upper part of esophagus and tracheoesophageal groove.

The surface of the liver was inspected and samples of grossly abnormal areas were sent for frozen section examination. The liver was elevated with an expandable fan retractor to expose the area of the gastroesophageal junction. The lesser curvature of the stomach was inspected for evidence of grossly abnormal lymph nodes. The lesser sac was entered using sharp dissection through the lesser omentum until the right crus was identified (Fig. 5 ). If imaging studies suggested enlarged nodes on the left crus, the peritoneum overlying the esophagus was opened and the left crus exposed. Biopsies of lymph nodes identified along the lesser curve were taken. Pulsations from the right gastric artery were usually visible caudally, and division of the omentum was stopped at this point.

The celiac axis was exposed by elevation of the lesser curve of the stomach near the gastroesophageal junction. The left gastric artery was identified by its pulsation as it projects straight up from the celiac axis and enters the posterior wall of the stomach. The left gastric artery was followed proximally to the origin of the celiac axis.

Ancillary procedures. In patients with a greater than 30-pound weight loss or those with preoperative severe dysphagia, a laparoscopic jejunostomy was constructed before the procedure was completed. The ligament of Treitz was located and a suitable segment of proximal jejunum, which reaches the abdominal wall at the site of the left upper quadrant port, was chosen. This was then exteriorized through a 3 to $4 \mathrm{~cm}$ incision created by enlargement of the operating port site. A standard Witzel feeding jejunostomy was constructed, and the jejunum was returned to the abdomen under laparoscopic visualization to ensure that no torsion was present and that adequate suture fixation to the posterior aspect of the abdominal wall was accomplished. Subcutaneous infusion ports for adjuvant chemotherapy were placed in all patients who agreed to undergo preoperative chemotherapy/ radiation therapy. A right subclavian or internal jugular single-lumen MediPort device (Bard Medsystems, Reading, Mass.) was placed under fluoroscopic guidance in these patients. In this way the anesthetic was used for diagnostic staging information and also for therapeutic

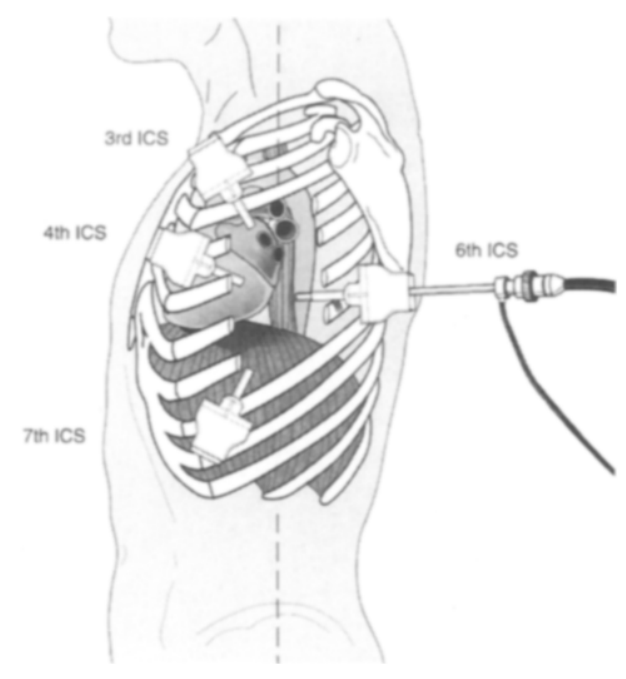

Fig. 3. Setup for left thoracoscopic anteroposterior node dissection. ICS, Intercostal space.

interventions in those patients who later received adjuvant chemotherapy and radiation therapy.

Technique of esophageal resection. Esophagectomy was performed with the use of combined abdominal, right chest, and neck incisions (McKewon) for resection of upper thoracic esophageal cancers. Resection via the left side of the chest or the right side of the chest and abdomen (Ivor Lewis) was done for midesophageal and gastroesophageal junction cancers. Transhiatal esophagectomy was generally avoided. This allowed for maximal sampling of mediastinal and abdominal lymph nodes. Follow-up therapy, including chemotherapy, radiation therapy, or both, was done independent of the staging procedure.

\section{Results}

Thoracoscopic lymph node staging was attempted in 45 consecutive patients with potentially resectable tumors and was successfully completed in 42 . In three patients attempts at thoracoscopy were aborted because of severe adhesions in the chest. There were no deaths. One patient had pneumonia in the postoperative period. There were no instances of bleeding or prolonged chest tube drainage. The mean operating room time was $2 \frac{1}{2} 2$ hours. Although esophagectomy could theoretically be undertaken at the same sitting, we routinely waited for permanent section pathology reports. At follow-up esophagectomy, adhesions were noted along the previous biopsy sites, but these did not complicate the procedure in any way. We routinely used two of the prior incisions in the thoracotomy and one for the chest tube at the time of esophagectomy. All esophagectomies were done often within 1 to 2 weeks of 


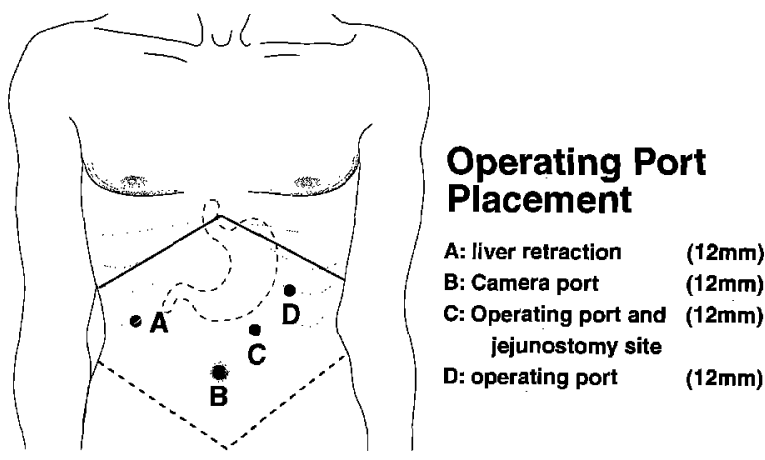

Fig. 4. Setup and port placement for laparoscopic lymph node staging.

staging or within 4 weeks after preoperative chemotherapy/radiation therapy for those patients treated on neoadjuvant protocols. The pathologic staging of these patients, based on thoracoscopic lymph node staging, was N0 in 39 patients and N1 in three patients. Of the 19 patients who underwent laparoscopic lymph node staging, the pathologic findings were normal celiac lymph nodes in 13 patients and diseased lymph nodes in six patients.

Among the 42 patients who underwent thoracoscopic staging, subsequent esophagectomy was accomplished in 30 patients; in 12 patients staging was done but resection was not. Among patients who did not undergo esophagectomy were seven who underwent successful thoracoscopic staging but refused esophageal resection and were treated by chemotherapy and/or radiotherapy only. In two patients pulmonary metastases were detected at thoracoscopic staging and they were treated with chemotherapy/radiotherapy only. In one patient malignant invasion of the trachea was detected at bronchoscopy. One patient had obvious intraperitoneal metastases at laparoscopy and therefore did not undergo thoracoscopic lymph node staging. One patient had severe portal hypertension, which complicated an attempt at laparotomy.

Esophageal resection was performed in 30 patients after thoracoscopic and/or laparoscopic staging. All 30 patients underwent thoracoscopic staging and 17 of these $30(60 \%)$ underwent laparoscopic staging. These patients form the basis for subsequent data analysis.

Pathologic findings after thoracoscopic staging in the 30 patients revealed N0 lymph node status in 28 patients and N1 in two patients (Fig. 6). Two of the 28 patients $(7 \%)$ with N0 disease were ultimately found to have paraesophageal lymph node involve-

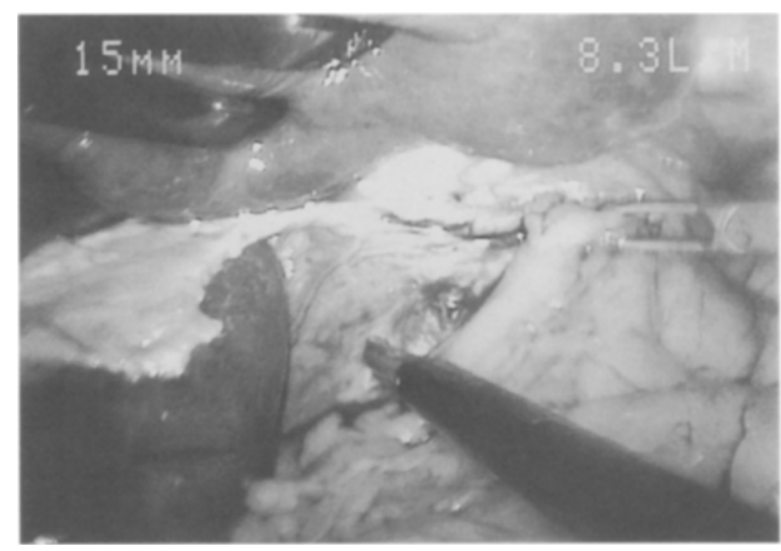

Fig. 5. The liver is retracted superiorly and laterally, and specimens of lesser curvature lymph nodes are retrieved as the lesser omentum is divided to the hiatal crus.

ment (N1) at final pathologic staging after esophageal resection. Thus their disease was understaged by thoracoscopy. In one of these cases a single parahiatal lymph node was diseased among more than a dozen nodes sampled. No parahiatal lymph node was diseased either by thoracoscopic or laparoscopic sampling. Two of 30 patients had N1 disease at thoracoscopic lymph node staging. One of these patients had no lymph node involvement (N0) at final pathologic staging after chemoradiation and the other patient had N1 disease at the time of resection. Thus thoracoscopic staging was accurate in detecting the presence of thoracic lymph node disease in 28 of 30 patients (93\% accuracy).

Seventeen patients underwent laparoscopic lymph node staging followed by esophagectomy (Fig. 7). Twelve patients had normal celiac lymph nodes and five had diseased celiac lymph nodes at laparoscopic staging. After esophagectomy, final pathologic staging in the 12 patients with $\mathrm{N} 0$ disease was N0 in 11 and diseased celiac lymph nodes in one patient. Final pathologic staging in the five with diseased lymph nodes at laparoscopic staging revealed diseased lymph nodes in two patients. Three patients were downstaged to N0 status after chemotherapy/radiotherapy. Thus laparoscopic lymph node staging was accurate in detecting lymph node metastases in 16 of 17 patients (94\%). The sensitivity, specificity, positive predictive value, and negative predictive values for thoracoscopy and laparoscopy are shown in Table I.

\section{Discussion}

If it were possible to obtain exact preoperative staging in esophageal cancer, patients could be 
PREOPERATIVE

TSLN

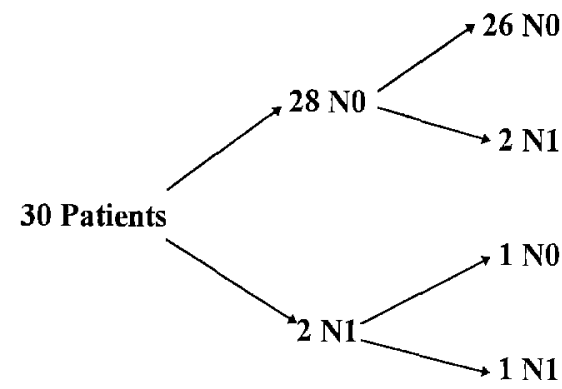

Fig. 6. Results of thoracoscopic lymph node (TSLN) staging compared with resection.

Table I. Sensitivity, specificity, positive predictive value, and negative predictive value of thoracoscopic (TSLN) and laparoscopic (LSLN) lymph node staging

\begin{tabular}{lcc} 
& TSLN & LSLN \\
\hline Sensitivity (\%) & 50 & 14 \\
Specificity (\%) & 100 & 100 \\
Positive predictive value (\%) & 100 & 100 \\
Negative predictive value (\%) & 93 & 92 \\
Accuracy (\%) & 93 & 94
\end{tabular}

separated prospectively into those likely to have residual local or lymphatic disease, who might be candidates for neoadjuvant therapy, and those believed likely to benefit from immediate curative resection. This would allocate adjuvant therapy to the appropriate patient populations. Unfortunately, the staging tools for esophageal cancer have not been precise and certainly lack the precision seen in the lung cancer.

Although earlier studies reported favorable results with CT, recent reports have been more circumspect about the accuracy of CT in determining the resectability of esophageal carcinoma. ${ }^{6}$ Limited literature exists on the role of MRI for esophageal carcinoma. ${ }^{7}$ Thus far, no significant benefit has been shown for MRI versus CT. All MRI studies have used only conventional $\mathrm{T} 1$ and $\mathrm{T} 2$ weighted spin-echo images. Gadolinium enhancement has not been used in staging esophageal cancer, although a pilot study is currently underway at our institution. ${ }^{8}$ EUS is a relatively new tool for evaluation of esophageal lesions. Recently, it has been used for staging and is highly accurate in detecting the depth of tumor invasion. ${ }^{9}$ In expert hands, EUS has an accuracy for predicting lymph node
PREOPERATIVE LSLN POST-RESECTION

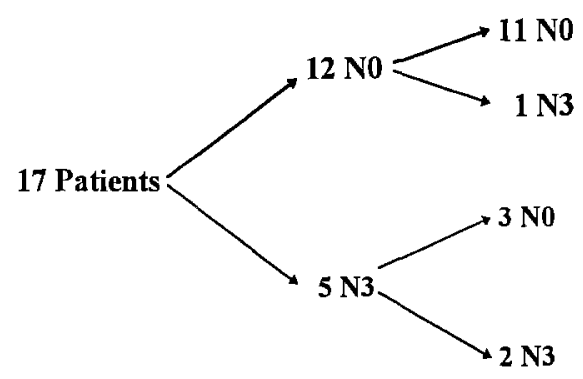

Fig. 7. Results of laparoscopic lymph node ( $L S L N)$ staging compared with resection.

status of $79 \%$ to $88 \%$. At present, EUS fails in a significant number of patients because of obstruction (26\% to $62 \%$ ). Unfortunately, in most of these studies, strict radiologic-surgical-pathologic correlation has been lacking.

Lymph node metastases commonly occur along the lesser curvature of the stomach and in the celiac axis. In a study by Akiyama and associates, ${ }^{10}$ spread to distant lymph nodes from all levels of esophageal cancer was noted including thoracic lymph node stations regardless of the level of tumor. Skinnner, Dowlatshahi, and DeMeester ${ }^{11}$ described the importance of lymph node staging in selecting the type of procedure performed for patients with esophageal cancer, emphasizing that extensive resections should be considered for patients with spread to periesophageal lymph nodes. In a recent study by Ellis, Watkins, and Krasna, ${ }^{12}$ the presence of lymph node metastases in esophageal cancer was noted as an independent predictor of prognosis. Preoperative surgical staging in esophageal cancer was first described by Murray, Wilcox, and Starek ${ }^{13}$ in 1977. In this study, mediastinoscopy and minilaparotomy were performed prospectively in 30 patients. In seven patients diseased lymph nodes were found in the mediastinum and in 16 patients diseased celiac lymph nodes were identified. The authors believed that lack of access to thoracic lymph nodes was one of the limiting factors. Thoracoscopy and laparoscopy are the most accurate lymph node staging modalities currently available. Although they do require a minimally invasive procedure, with experience the staging and access/nutrition procedures can all be done in 2 hours.

Although laparoscopy has been used for staging of intraabdominal malignant tumors since the mid1970s in some centers, little information exists re- 
garding staging of esophageal cancer. ${ }^{14-18}$ It is a relatively easy matter to inspect the parietal peritoneum and surface of the liver and other viscera. However, the instruments and surgical techniques necessary for safe dissection in the lesser sac, near the aorta, for the harvest of regional esophageal lymph nodes have only recently been developed. Previous reports of laparoscopy for staging of esophageal cancer have detected intraabdominal disease in $16 \%$ to $24 \%$ of patients. ${ }^{15,18}$ Accuracy in the detection of hepatic metastases from esophageal cancer is as high as $96 \%$, and laparoscopy has proved superior to ultrasound or CT for this application in prospective trials. ${ }^{18}$

In two of 30 patients (7\%) the disease was understaged (missed N1 lesions) with respect to thoracic lymph node involvement by thoracoscopy. Although we assume that the thoracoscope missed these N1 lesions, it is possible that there was "progression of disease" in the interim between staging and resection. In one of $30(3 \%)$ patients the disease was downstaged (N1 to N0) after preoperative chemotherapy/radiotherapy. The status of three patients with diseased celiac lymph nodes at laparoscopy was downstaged to N0 at resection after preoperative chemoradiation. Thoracoscopy was accurate in detecting the presence of lymph node metastases in 28 of 30 patients (93\%) and laparoscopy was accurate in 16 of 17 patients (94\%). Before our use of laparoscopy, we missed celiac metastases in two patients who underwent esophageal resection after thoracoscopic lymph node staging. In this series, five of 18 patients $(33 \%)$ had unsuspected celiac axis lymph node involvement according to preoperative CT. Since routine laparoscopic staging was begun, a diseased celiac lymph node has been missed in only one patient. Thoracoscopic lymph node staging also correctly predicted locally unresectable disease in three patients and pulmonary metastases in two patients. In two patients, T4 lesions identified at surgery were missed by thoracoscopy.

In patients with locally advanced non-small-cell carcinoma of the lung, the roles of surgery and chemotherapy/radiotherapy depend on accurate preresection staging with both noninvasive and invasive tests. The use of mediastinoscopy in preoperative staging for lung, cancer was shown to be highly efficacious by Pearson and associates. ${ }^{19}$ The usefulness of this technique suggests a possible role for preoperative surgical staging in esophageal cancer, to delineate which patients have advanced disease and which patients will in fact have early local disease.
Thoracoscopy and laparoscopy have the potential to achieve accurate surgical staging of lymph nodes with minimal morbidity. The goal of this study was to define their accuracy and usefulness in staging esophageal cancer. Preresectional surgical staging of mediastinal and abdominal lymph nodes for esophageal cancer may prove to be the most effective method of accurately stratifying patients before the start of adjuvant therapy.

\section{Conclusions}

These results suggest that preoperative thoracoscopic/laparoscopic staging can be used to accurately assess thoracic and abdominal lymph node status in esophageal cancer. Thoracoscopic/laparoscopic staging shows promise as a tool to identify those patients with advanced locoregional disease who may benefit from preoperative neoadjuvant therapy. In this way, selected subgroups of patients may achieve higher rates of success vis à vis survival and recurrence, as we have seen in subgroups of lung cancer.

\section{REFERENCES}

1. Orringer MB, Forastiere AA, Perez-Tamayo C, et al. Chemotherapy and radiation therapy before transhiatal esophagectomy for esophageal carcinoma. Ann Thorac Surg 1990; 119:348-55.

2. Fiocco M, Krasna MJ. Thoracoscopic lymph node dissection in the staging of esophageal disease. J Laparoendosc Surg 1992;2:111-5.

3. Krasna MJ, McLaughlin JS. Thoracoscopic lymph node staging for esophageal Cancer. Ann Thorac Surg 1993;56: 671-4.

4. Casson AG, Inculet RI, Zankowicz N, et al. Lymph node mapping for resectable carcinoma of the esophagus: a guide for thoracic surgeons. Princeton, New Jersey: Bristol-Myers Squibb Company, 1992.

5. Wolfer RS, Krasna MJ, Hasnain JU, et al. Hemodynamic effects of carbon dioxide insufflation during thoracoscopy. Ann Thorac Surg 1994;58:404-8.

6. Lefor AT, Bredenberg CE, Kellman RM, et al. Multiple malignancies of the lung and head and neck: second primary tumor or metastasis? Arch Surg 1986;121:265-70.

7. Maas R, Nicholas V, Grimm H, et al. MRI of esophageal carcinoma with ECG garing at 1.5 Tesla. Ferguson MN, Little AG, Skinner DB, eds. Diseases of the esophagus. Mount Kisko, New York: Futura, 1990:145-55.

8. Templeton PA, Krasna MJ. Use of gadolinium enhanced MRI to evaluate for airway invasion in patients with esophageal carcinoma [Abstract]. Radiological Society of North America, 1994.

9. Rice TW, Boyce GA, Sivall MV. Esophageal ultrasound and the preoperative staging of carcinoma of the esophagus. $J$ Thorac Cardiovasc Surg 1991;101:536-43.

10. Akiyama $H$, Tsurumaru M, Kawamura $T$, et al. Principles of surgical treatment for carcinoma of the esophagus. Ann Surg 1981;194:438-46. 
11. Skinner D, Dowlatshahi K, DeMeester T. Potentially curable cancer of the esophagus. Cancer 1982;50:2571-5.

12. Ellis H, Watkins E, Krasna M. Staging of carcinoma of the esophagus: a comparison of different staging criteria. J Surg Oncol 1993,52:231.

13. Murray G, Wilcox B, Starek P. The assessment of operability of esophageal carcinoma. Ann Thorac Surg 1977;23:393-9.

14. Cuschieri A. Diagnosis and staging of tumors by laparoscopy. Semin Laparosc Surg 1994;1:3-12.

15. Dagnini G, Caldironi MW, Marin G, et al. Laparoscopy in abdominal staging of esophageal carcinoma: report of 369 cases. Gastrointest Endosc 1986;32:400-2.

16. Shandall A, Johnson C. Laparoscopy or scanning in oesophageal and gastric carcinoma? Br J Surg 1985;72-449-51.

17. Watt I, Stewart I, Anderson D, et al. Laparoscopy, ultrasound and computed tomography in cancer of the oesophagus and gastric cardia: a prospective comparison for detecting intra-abdominal metastases. Br J Surg 1989;76:1036-9.

18. Lightdale CJ. Laparoscopy for cancer staging. Endoscopy 1992;24:682-6.

19. Pearson FG, Nelems JM, Henderson RD, et al. The role of mediastinoscopy in the selection of treatment for bronchial carcinoma with involvement of superior mediastinal lymph nodes. J Thorac Cardiovasc Surg 1972;64:382-90.

\section{Discussion}

Dr. David J. Sugarbaker (Boston, Mass.). It appears clear that until we are able to adequately stage esophageal cancer before starting therapy, we will not be able to effectively assess the effect of specific induction therapy regimens on the tumor. Our group at the Brigham has been actively involved in the application of thoracoscopic and laparoscopic staging to esophageal cancer. It is clear from reports of patients having surgical resection for esophageal cancer that the two groups of patients at high risk are those with $\mathrm{T} 3$ lesions, that is, tumors penetrating the muscularis, and those with $\mathrm{T} 1$ or $\mathrm{T} 2$ lesions that have N1 disease.

These two high-risk subgroups, those with T3 lesions regardless of $\mathrm{N}$ and those patients with $\mathrm{T} 1$ or $\mathrm{T} 2$ lesions with $\mathrm{N} 1$ disease, constitute excellent subgroups of esophageal cancer to identify and to which to apply these new staging techniques before therapy. The uniformly poor outcome of these two high-risk groups with surgical therapy alone makes them the ideal subcategory of patients to identify and justifies the additional morbidity of neoadjuvant, induction therapy. By pathologically documenting the extent of disease before therapy, we may be able to develop better regimens based on the pathologic readout at the time of surgical resection.

With the changing epidemiology of esophageal cancer from the traditional larger squamous lesions to smaller adenocarcinomas, it may be that in some patients with superficial lesions originating in a Barrett's esophagus, the added morbidity of induction therapy may be unjustified. Therefore, this staging approach is of additional value to the patient who has a $\mathrm{T} 1$ lesion by assessing the involvement of lymph nodes before the decision to surgically resect the tumor or to proceed with an induction neoadjuvant chemotherapy/radiotherapy protocol. A reliable technique, in this setting, would be beneficial to a large number of patients who have very superficial adenocarcinomas arising usually in a Barrett's esophagus.

Two factors will push the need for adequate staging before therapy. The first is the rising popularity of induction therapy in esophageal cancer, and the second is the changing epidemiology from larger squamous lesions to smaller more superficial adenocarcinomas. It is in this setting that the work of Dr. Krasna and his coworkers, I believe, will be of significance.

I have the following questions for Dr. Krasna. In our institution a significant learning curve has been incurred, particularly in the use of laparoscopic biopsy of the celiac nodes. Would you please comment on the learning curve in your institution and ways in which it may be minimized? The second question deals with additional nutritional support to be used during and after neoadjuvant therapy. Have you been able to coordinate these efforts with thoracoscopic staging in patients with esophageal cancer? Third, please comment on the use of EUS to identify the high-risk T3 lesions. How do you coordinate the use of EUS with thoracoscopic and laparoscopic staging of esophageal lesions to maximize the identification of patients with either T3 or N1 disease. Finally, please comment on the additional value that thoracoscopy has in identifying pleural implants or T4 extension of tumor in the evaluation of these patients during the thoracoscopic exploration.

Dr. Krasna. Regarding the learning curve, I learned to do the laparoscopic staging with Dr. Jack Flowers, who is our laparoscopic expert. I would encourage anybody undertaking laparoscopic staging, if you have not done laparoscopy in the past, to involve your gastrointestinal laparoscopic surgeon early on. The first 10 or so cases he did with me, and now I generally do them myself.

In terms of nutritional support, we do use the same anesthesia to almost routinely now place either a laparoscopic jejunostomy or a MediPort device, sometimes both, since many of these patients undergo adjuvant therapy.

I have not been using EUS to localize the lesions, although I understand from Dr. Reed in South Carolina that there is some promising data on the use of some of the new EUS-directed needle biopsies.

Regarding pleural and T4 lesions, we did not find any malignant pleural effusions or pleural implants. We did, however, find that thoracoscopy allowed us to avoid an unnecessary thoracotomy in three patients who had T4 tumors. That was after we learned the hard way on the two patients in whom thoracoscopic staging was used. They were inappropriately subjected to a resection, and tumor was found invading the airway.

Dr. Jack A. Roth (Houston, Tex.). One of the biggest barriers to evaluating new treatment modalities is accurate staging of carcinoma of the esophagus. It should be emphasized that induction therapy and the use of preoperative radiation and chemotherapy are certainly not standard treatments for this disease. It is important to evaluate these modalities in prospective studies, and this type of staging evaluation may contribute to the accuracy of those studies.

I have one concern about this type of approach. In a number of patients who have undergone thoracoscopic evaluation for primary or metastatic malignancy, we have 
noted some seeding of the thoracoscopic tract and incision, associated with local recurrence, which might be attributed to the thoracoscopic resection. This does not seem to be related to the use of thoracoports or endobags. Despite very careful technique, we still find these local recurrences in a disturbingly high percentage of patients. Have you noticed these in any of your patients who have undergone this type of evaluation?

Dr. Krasna. We have not had any cases of either seeding or local recurrence. We do routinely use thoracoports. We never pull a lymph node biopsy specimen, even a small lesion, through the open wounds, because we believe that the port will allow some protection. Obviously if a pulmonary lesion is identified, it is removed with an endobag of some sort. We would never bring the lesion directly out. Although the laparoscopic literature reports an apparent propensity for the tumors to recur at the
Iaparoscopic port sites, we have not yet seen this in the more than 350 thoracoscopies that we have done.

Dr. Nasser Altorki (New York, N.Y.). I just reviewed almost 100 cases in our institution, and the incidence of nodal metastasis was about $70 \%$. In your data it is almost $90 \%$ node negative. I wonder whether that reflects a low sensitivity for detecting those nodes. What do you do if you get a diseased node on the thoracoscopy? How has that affected your management of the patient?

Dr. Krasna. We were quite concerned at first, because many reports have stressed $30 \%$ to $70 \%$ incidences of diseased nodes. I should stress that all of these patients underwent formal esophagectomy with lymph node sampling-not the radical dissection as Dr. Skinner proposes, but a very exhaustive lymph node sampling from the apex to the hiatus. Thus, although the incidence was low overall, we do not believe this represents a false positive result. 